\title{
Angiolipoma renal con extensión tumoral a vena cava inferior y aurícula derecha*
}

\section{Left kidney angiomyolipoma, spreading to the renal vein, inferior vena cava and involving the heart. Report of one case}

\author{
Drs. FRANCO INNOCENTI C. ${ }^{1,6}$, EMILIO ALARCÓN C. ${ }^{2}$, ESTEBAN ARIAS O. ${ }^{3}$, ALECK STOCKINS L. ${ }^{2}$ \\ ROBERTO SÁNCHEZ U. ${ }^{4}$, JAIME MADARIAGA B. ${ }^{5}$, RODRIGO TORRES-QUEVEDO Q.6 , ALS. FELIPE \\ CABRERA E.7, RAMÓN DÍAZ J.7

\begin{abstract}
1 Unidad de Trasplante, ${ }^{2}$ Unidad de Cirugía Cardiotorácica, ${ }^{3}$ Servicio de Urología, Hospital Clínico Regional de Concepción; ${ }^{4}$ Departamento de Cirugía, Universidad de Concepción, ${ }^{5}$ Servicio de Anatomía Patológica, Hospital Regional de Concepción. ${ }^{6}$ Servicio de Cirugía, Clínica Sanatorio Alemán de Concepción. ${ }^{7}$ Alumnos Facultad de
\end{abstract} \\ Medicina, Universidad de Concepción. Concepción, Chile
}

\begin{abstract}
RESUMEN
El angiomiolipoma renal es un tumor benigno mesenquimático. Constituyen sólo del 2 al $6 \%$ de los tumores renales. Existen alrededor de 12 casos reportados con invasión a las venas renales y la cava inferior. En menos de 5, hay extensión de trombo tumoral hasta aurícula derecha. Objetivo: Presentar el caso clínico, manejo y evolución de una paciente con un angiomiolipoma renal con extensión tumoral a vena renal y cava inferior y que compromete la cavidad auricular derecha casi en su totalidad. Paciente y método: 50 años, sexo femenino con hallazgo del tumor por un ultrasonografía abdominal motivada por el estudio de síntomas digestivos de origen probablemente funcional. Se confirma el diagnóstico con TAC y ecocardiograma doppler. Se realiza una cirugía combinada, abdominal y esternotómica, con la ayuda de un by pass aortopulmonar. Resultados: Nefrectomía izquierda, liberación intravascular del tumor que se empuja por cava inferior y se extrae en block por la aurícula derecha. Evoluciona inicialmente en forma satisfactoria, pero desarrolla distress respiratorio con angio TAC de tórax negativo para TEP a las $48 \mathrm{~h}$ de la cirugía. Se inicia anticoagulación empírica con HBPM y encontrándose extubada y en buenas condiciones hace un hemoperitoneo el día 14, encontrándose hemorragia en napa en los sitios de disección previa. De alta a los 21 días. El seguimiento alejado a los 2 años revela una hernia incisional, reparada sin incidentes, y sin otras complicaciones ni signos de recidiva de patología original. Conclusión: El manejo de equipo multidisciplinario nos permitió ayudar exitosamente a esta paciente con patología rara y compleja.
\end{abstract}

\section{PALABRAS CLAVE: Angiomiolipoma renal, aurícula derecha.}

\section{SUMMARY}

Renal angiomyolipoma is an uncommon benign tumor of mesenchymal origin. In less than five of 12 cases reported with renal vein and inferior vena cava involvement the thrombus extends to the right atrium.

*Recibido el 1 de Agosto de 2007 y aceptado para publicación el 1 de Septiembre de 2007.

Correspondencia: Dr. Franco Innocenti

Alto Llacolén 776, Idahue, San Pedro de La Paz, Concepción, Chile

Fax 041-2290550

e-mail: finnocenti@sanatorioaleman.cl 
We report a 50 years old female with a left kidney angiomyolipoma, spreading to the renal vein, inferior vena cava and involving the heart, invading the right atrium almost completely. The tumor was found during a study for abdominal pain. She underwent combined abdominal and cardiac surgery with pulmonary bypass. A left nephrectomy, cavotomy and intravascular dissection of the tumor were performed. The intravascular mass was pushed from abdomen and pulled out through the right atrium. Forty eight hours after surgery, she developed respiratory distress. A chest angio CT scan negative for pulmonary thromboembolism. However, anticoagulation with low molecular weight heparin was started due to the high risk for thromboembolism. She recovered, but 14 days after the original surgery, presented a massive hemoperitoneum. She was operated again, finding a diffuse oozing from the sites of previous dissection. The dose of anticoagulation was lowered, with a good postoperative evolution, being discharged 21 days later. After two years of follow up, she developed an incisional hernia that is repaired.

\section{KEY WORDS: Angiomyolipoma, inferior vena cava, thromboembolism.}

\section{INTRODUCCIÓN}

El angiomiolipoma renal es un tumor poco frecuente de origen mesenquimático, con breves referencias en los textos clásicos de anatomía patológica. Su estructura la forma un componente de músculo liso, otro vascular y otro graso. Se presenta en forma esporádica o en asociación con una esclerosis tuberosa. Es de naturaleza benigna'. Sin embargo, en raras ocasiones puede tener una evolución de curso potencialmente maligno, invadiendo la vena renal, la $\mathrm{VCl}$ e incluso la aurícula derecha ${ }^{2-7}$. Hay pocos casos reportados con un trombo tumoral intracardíaco. Reportamos el caso de una paciente con angiomiolipoma con extensión del tumor hasta la aurícula derecha.

\section{CASO CLÍNICO}

Paciente de sexo femenino, 50 años de edad, cuyo motivo de consulta fue por cólicos y distensión abdominal frecuentes, sin otra historia pasada significativa. Dentro de sus antecedentes médicos sólo se encuentra un síndrome bronquial obstructivo documentado con espirometría y que alivia con broncodilatadores. Sin signos ni síntomas de insuficiencia cardiaca, sin edema de extremidades. Durante su estudio con ultrasonografía abdominal se pesquisa el hallazgo tumoral, por lo que se completa con tomografía axial computada y ecocardiograma, los que confirman que el compromiso se extiende hasta la aurícula derecha, sin insuficiencia tricuspídea ni compromiso de la función cardiaca hasta ese momento (Figuras 1y 2).

Se realiza una cirugía combinada, con esternotomía media y laparotomía subcostal bilateral con prologación xifoídea. Se moviliza el colon derecho e intestino delgado en su totalidad con maniobra de Catell, disecándose la vena cava inferior desde la bifurcación de las venas ilíacas por cau-

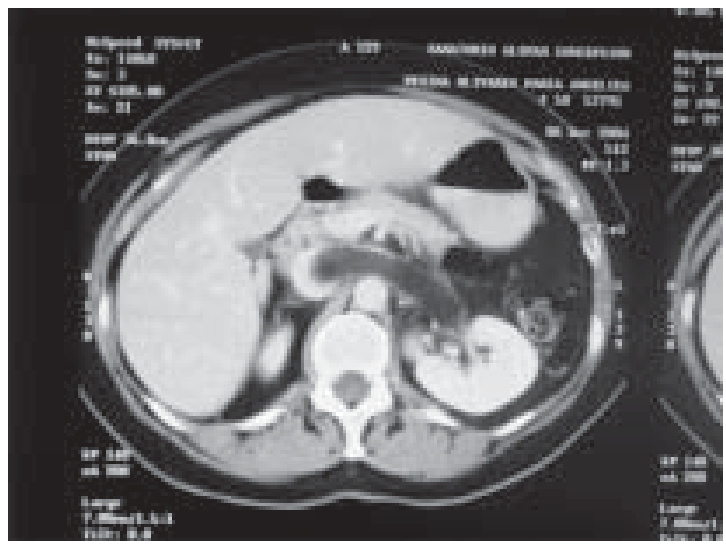

Figura 1. Tomografía axial computada (TAC) que revela riñón izquierdo con compromiso tumoral intravascular que ocupa completamente la vena renal y comienza a ocupar el lumen de la vena cava inferior (VCI).

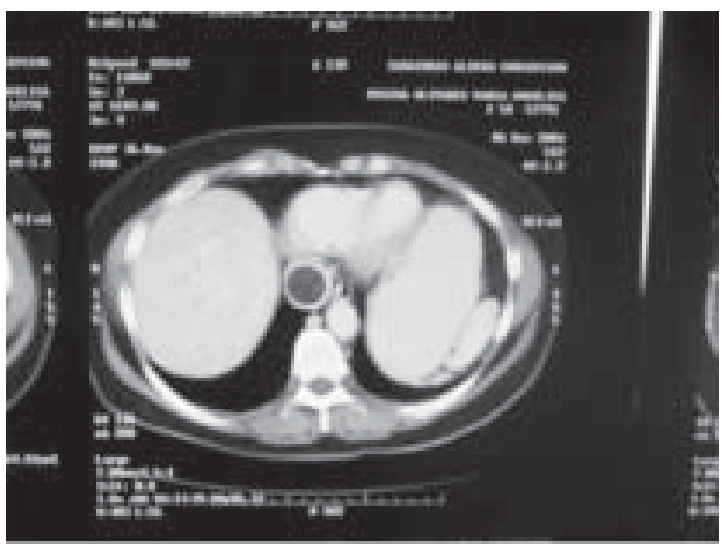

Figura 2. Tomografía axial computada en la que se observa el tumor en el lumen de la vena cava retrohepática y permite observar gran ocupación del lumen de la VCI por el tumor, que se extiende desde el riñón izquierdo hacia cefálica. 
dal hasta por sobre la desembocadura de la vena renal izquierda, identificando la arteria mesentérica superior en su raíz. Se libera el riñón izquierdo desde lateral, conservando la fascia de Gerota. Se ligan y seccionan ramas venosas suprarrenales y gonadales izquierdas (Figura 3).

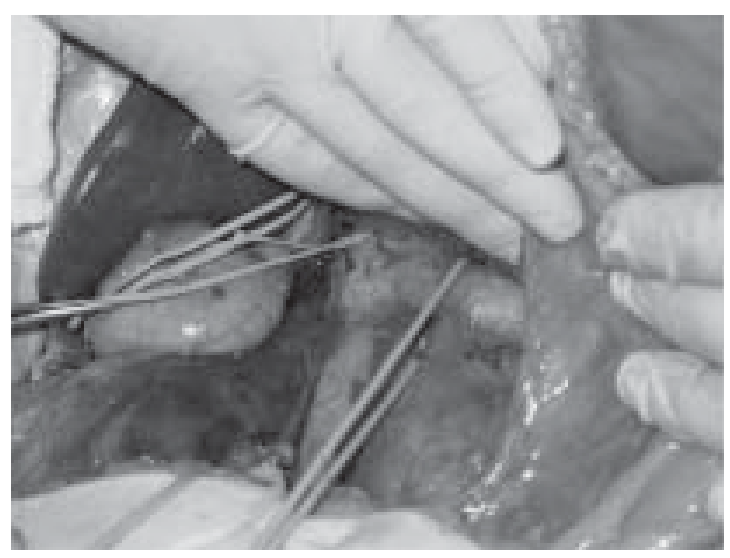

Figura 3. Disección de venas renal izquierda y $\mathrm{VCI}$ subhepática, ambas en vessel loops.

Se moviliza el hígado seccionando el ligamento triangular izquierdo y el peritoneo entre el segmento I y la vena cava retrohepática, ligando y seccionando un par de ramas VCl-segmento I. Se tiene completo acceso a la vena cava retrohepática por un acceso lateral izquierdo.

Se prepara la porta hepatis para una maniobra de Pringle. Se efectúa auriculotomía derecha y se entra en circulación extracorpórea (CEC), al tiempo que se clampea la $\mathrm{VCI}$ distal y realiza la maniobra de Pringle con el objeto de reducir el débito hacia la aurícula desde la VCl. Se liga la arteria renal izquierda y realiza venotomía renal parcial, sólo en su cara anterior, a $2 \mathrm{~cm}$ del hilio (Figura 4). La vena renal tiene aquí un diámetro de $2.5 \mathrm{~cm}$. Se divide el tumor en esta zona y se diseca digitalmente hasta la vena cava, empujándose el tumor hacia la aurícula derecha, por donde es completamente extraído. Se liga vena renal hacia la cava, se sueltan clamps de $\mathrm{VCl}$ y Pringle y se evidencia un excelente flujo por la VCI hacia la aurícula. Se cierra la auriculotomía y, una vez recuperada temperatura, se sale de CEC sin incidentes. Se completa nefrectomía izquierda (Figura 5) y se restituye anatomía de colon e intestino. Se finaliza la cirugía revisando la hemostasia y cerrando tanto la esternotomía como la laparotomía en forma habitual.

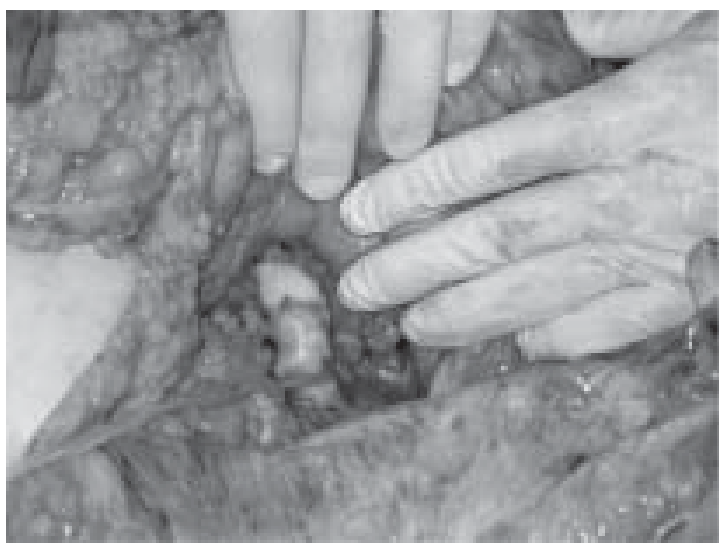

Figura 4. Se observa la vena renal y un segmento del tumor seccionados en su cabo periférico rodeados por cinta umbilical, una vez disecado y empujado el segmento central a través de la $\mathrm{VCI}$ y ligado el muñón de la vena renal a $2 \mathrm{~cm}$ de la $\mathrm{VCl}$.

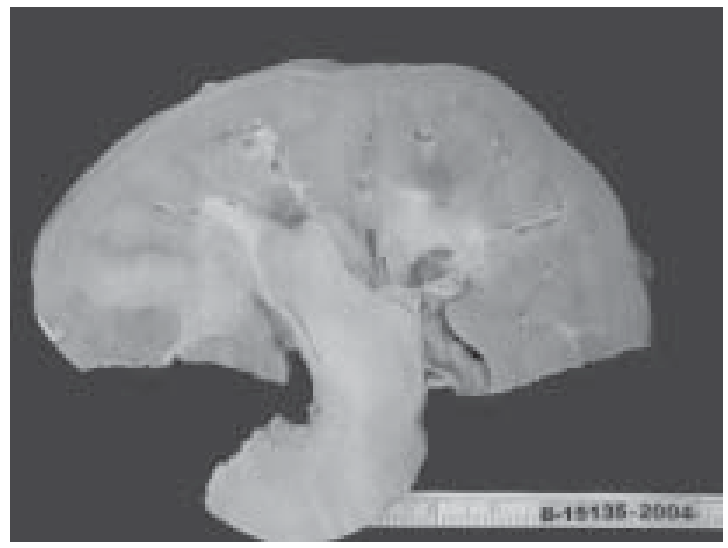

Figura 5. Espécimen parcial de anatomía patológica que muestra el compromiso corticomedular del seno renal izquierdo y el crecimiento tumoral endovascular.

La paciente evoluciona inicialmente en forma satisfactoria, pero desarrolla distress respiratorio con angio TAC de tórax negativo para TEP a las $48 \mathrm{~h}$ de la cirugía. Se comienza una anticoagulación empírica con heparina de bajo peso molecular. Ya en buenas condiciones hace un hemoperitoneo al día 14, encontrándose una hemorragia en napa en los sitios de disección previa. Rápido control sin nuevo sangrado, se suspende y luego reinicia con menor dosis el anticoagulante De alta a los 21 días. En el seguimiento alejado, continúa en control broncopulmonar por síndrome bronquial obstructivo, sin evidencias de enfermedad residual (Figura 6). Hace una hernia incisional que se repara a los 2 años de la cirugía original, sin incidentes. 


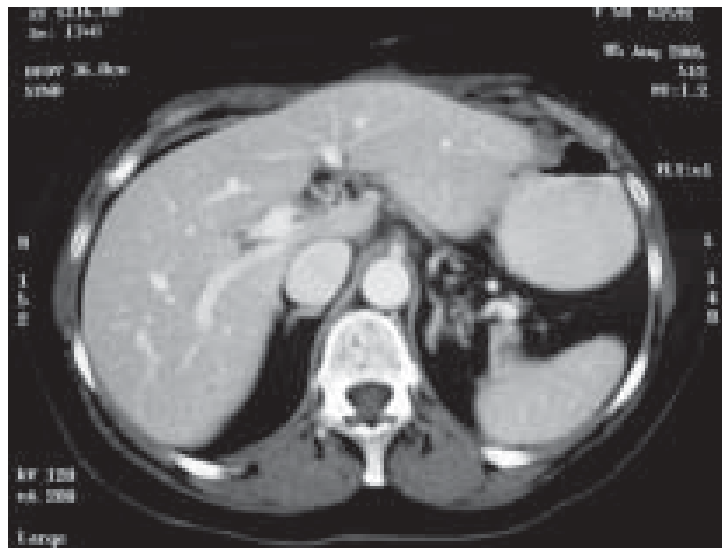

Figura 6. Tomografía axial computada 2 años post cirugía, en la que se observa la vena cava libre. Resto del estudio de imágenes no mostró evidencia de enfermedad residual o recurrencia.

\section{DISCUSIÓN}

El angiomiolipoma renal es un tumor benigno poco frecuente. Su manejo en general depende de su tamaño. Cuando es menor o igual a $4 \mathrm{~cm}$ se preconiza observarlos, con seguimiento por imágenes con una periodicidad que varía según la literatura médica. Sin embargo cuando es mayor de ese tamaño, o en raras ocasiones compromete la vena renal, cava o incluso el corazón se prefiere la cirugía ${ }^{2-7}$. En el caso que nos ocupa, aunque el tumor llegaba a la aurícula derecha, la paciente no presentaba síntomas ni signos cardiovasculares. La decisión de la cirugía se basaba en el riesgo de falla cardiovascular, trombosis o embolia, o de congestión venosa no sólo de las extremidades sino también de los órganos intraabdominales (congestión esplácnica, síndrome de Budd-Chiari, etc.). Este caso nos recuerda su rareza. También nos confirma que cuando se suman las experiencias de distintos equipos en un hospital terciario de nuestro sistema público de salud, es posible ayudar a estos pacientes cambiando su expectativa de sobrevida y con excelente calidad de vida.

\section{REFERENCIAS}

1. Rennke H. The kidney. En: Cotran R, Kumar V, Collins T, ed. Robbins Pathologic basis of disease. Philadelphia: Saunders, 1999: 991.

2. Gamé X, Soulié M, Moussouni S, Roux D, Escorrrou $G$, Chevreau $C$ et al. Renal angiomyolipoma associated with rapid enlargement and inferior vena caval tumor thrombus. The J of Urol 2003; 170: 918919.

3. Gotoh A, Gohji K, Fujisawa M, Okada H, Arakawa S, Hanioka $\mathrm{K}$ et al. Renal angiomyolipoma associated with inferior vena caval tumour thrombus. $\mathrm{Br} \mathrm{J}$ Urol 1998; 81: 773-774.

4. Rubio-Briones J, Palou J, Salvador J, Minino L, Garcia J, Parada R et al. Incidentally detected renal angiomyolipoma with tumour thrombus into the inferior vena cava. Scan J Urol Nephrol 1996; 31:189192.

5. Toda F, Okuda H, Kondo N, Iguchi Y, Ryoji O, Ito F et al. Renal angiomyolipoma with a tumor thrombus extending into the right atrium-a case report. Nippon Hinyokika Gakkai Zasshi 1999; 8:145-149.

6. Davydov M, Matveev V, Lukiachenko A, Kudashev B, Petrovichev N. Renal angiomyolipoma extending into the right atrium. Urol Int 2001; 67: 168-169.

7. Ito H, Nakashima S, Toma S, Misaki T. Renal angiomyolipoma associated with inferior vena caval and right atrial thrombus. The J of Urol 1999; 162: 13711372. 